\title{
Fibromyalgie-Behandlung: Repetitive transkranielle Magnetstimulation überprüft
}

\author{
Guinot $M$ et al. Effects of repetitive transcranial \\ magnetic stimulation and multicomponent \\ therapy in patients with fibromyalgia: a \\ randomized controlled trial. Arthritis care \& \\ research 2021; 73: 449-458. doi:10.1002/ \\ acr.24118
}

Für chronische Schmerzen bei Fibromyalgie (FM) ist zum Teil eine Sensibilisierung des Zentralnervensystems mit veränderter Schmerzmodulation verantwortlich. Behandelt wird die FM meist mit einer Kombination aus verschiedenen Therapiekomponenten. Forscherinnen und Forscher in Grenoble haben nun die repetitive transkranielle Magnetstimulation (rTMS) eine nichtinvasive Hirnstimulationstechnik, auf ihre Wirksamkeit bei FM untersucht.

Die Multikomponententherapie (MT) bei FM beinhaltet Patientenaufklärung, kognitive Verhaltenstherapie und/oder Bewe- 
gungstherapie (v. a. Schwimmtherapie und aerobe Rekonditionierung). Die MT führt zu einer verbesserten Lebensqualität und zu einer leichten bis moderaten Verbesserung der Fibromyalgie-Symptome. Die auf der Kopfhaut applizierte rTMS erzeugt ein elektromagnetisches Feld, das die Erregbarkeit von kortikalen und tiefen Hirnarealen moduliert. Sie wird bereits bei verschiedenen neurologischen und psychiatrischen $\mathrm{Er}$ krankungen eingesetzt und ist für die Behandlung von Depressionen zugelassen. Die Forscherinnen und Forscher der Studie vermuteten, dass eine Modulation absteigender Nervenbahnen und des rechten limbischen Areals analgetische Effekte bei FM haben könnte. Neuere Metaanalysen beschreiben bereits, dass die rTMS Schmerzen bei Patienten mit chronischem Schmerzsyndrom oder FM signifikant reduzieren kann.

Die französischen Forscher untersuchten den Effekt der rTMS auf Schmerzen bei FMPatienten, die parallel eine MT durchführten. 39 FM-Patienten mit Schmerzwerten auf einer visuellen Analogskala (VAS) von $\geq 40 \mathrm{~mm}$ wurden in die Studie eingeschlossen. Die Patienten durchliefen ein MT-Programm über einen Zeitraum von 12 Wochen (3 Sitzungen pro Woche, Kombination aus Aerobic-Training, Wassergymnastik und Entspannung). Darüber hinaus wurden die Patienten zufällig randomisiert zu:

- MT und aktive rTMS (hochfrequent, primärer motorischer Kortex M1) über einen Zeitraum von 12 Wochen (1-2 Sitzungen pro Woche; $\mathrm{n}=18$; mittleres Alter $=46,5 ; 100 \%$ weiblich) oder

- MT und Schein-rTMS (Simulation ohne elektromagnetisches Feld; $\mathrm{n}=19$; mittleres Alter $=42,8 ; 79 \%$ weiblich).

Ergebnismessungen wurden zu Beginn, in Woche 14 und 6 Monate (Woche 40) nach Abschluss des Programms durchgeführt. Von primärem Interesse war die Schmerzreduktion, gemessen anhand des wöchentlichen Mittelwertes der selbstberichteten Schmerzstärke. Von sekundärem Interesse waren kardiorespiratorische Fitness, kardiale autonome Adaptionen und FM-Ergebnisse (gemessen mit Skalen für FM-Werte, Depression, Schlafeffizienz und Schmerzkatastrophisierung).

\section{Ergebnisse}

- Die Reduktion des Wochenmittelwerts der täglich berichteten Schmerzen unterschied sich nicht signifikant zwischen den Gruppen.

- Die Schmerz-VAS-Werte sowie die kardiorespiratorische Fitness, Lebensqualität, Depression und Katastrophisierung verbesserten sich in Woche 14 signifikant bei beiden Gruppen und blieben bis Woche 40 stabil. Die MT allein war demnach ausreichend, um Schmerzen zu modulieren und die Lebensqualität zu verbessern.

- Kardiale autonome Adaption und Schlafeffizienz veränderten sich in keiner der beiden Gruppen statistisch signifikant.

\section{FAZIT}

Laut Autoren zeigen die Daten, dass die repetitive transkranielle Magnetstimulation in Verbindung mit MT keine größere Schmerzreduktion als MT allein bei Patienten mit schwerer Fibromyalgie bewirkt. Eine aktualisierte Metaanalyse spreche ebenso gegen eine Wirkung der rTMS hinsichtlich Schmerzen und Lebensqualität. Einschränkung sei, dass ein Placeboeffekt der hier angewendeten rTMS-Simulation nicht ausgeschlossen werden könne. Außerdem mangle es der Studie an statistischer Power.

Annkatrin Wagner, Stuttgart 